KENT D. LERCH

\title{
Justitia im Bett des Prokrustes \\ Sinn und Unsinn der linguistischen Analyse von Rechtstexten
}

\section{Die Sprache und das Außersprachliche}

Die Sprachlichkeit des Rechts ist einer der wenigen Gegenstände, denen sowohl Sprach- als auch Rechtswissenschaft seit langem großes Interesse entgegengebracht haben. So selten sich die beiden Disziplinen auch gegenseitig wahrnehmen und beeinflussen mögen, sind sie sich doch immer darin einig gewesen, dass zwischen Recht und Sprache ein enger Zusammenhang bestehe, den es zu ergründen gelte. Damit ist indes nicht nur gemeint, dass alle Rechtshandlungen Sprachhandlungen sind und sich Rechtsarbeit als Arbeit in, an und mit Sprache vollzieht; es wird auch danach gefragt, ob die allgemeinen Bedingungen der Sprache, an die das Recht gebunden ist, nicht in die Eigenart des Rechts selbst eingreifen, ob nicht zwischen Recht und Sprache eine enge Strukturverwandtschaft bestehe.

Anregungen, sprachwissenschaftliche und rechtswissenschaftliche Kompetenz gleichberechtigt heranzuziehen, um zu Aussagen grundsätzlicher Natur über den Zusammenhang zwischen Recht und Sprache zu gelangen, werden daher in der Rechtswissenschaft auf Resonanz treffen; die Erwartungen an die Sprachwissenschaft sind hoch und gehen so weit, dass schon eine "Rechtstheorie als Sprachtheorie des Rechts" (Arthur Kaufmann) gefordert wurde. Genährt werden diese Erwartungen noch durch die Zuversicht, mit der sich viele Linguisten anschicken, das Verhältnis von Sprache und Recht zu erhellen. Ob die Sprachwissenschaft dazu aber tatsächlich in der Lage ist, kann bezweifelt werden: vielmehr scheint es, dass die Linguistik derzeit denkbar schlecht für diese Aufgabe gerüstet ist.

Blickt man auf die Entwicklung der modernen Linguistik, so ist es ganz verständlich, dass sie eine Wissenschaft sein wollte, die nur "Sprache an sich" ergründen sollte: In den frühen Jahren fürchtete sie mit Recht, von den Nachbarwissenschaften vereinnahmt zu werden, etwa 
von der Soziologie, Psychologie, Anthropologie, Philosophie oder Literaturwissenschaft, und die begeisterte, aber leichtgläubige Akzeptanz der Saussure'schen Dichotomisierung von langue und parole ist am einfachsten durch den Wunsch der Linguisten zu erklären, sich in einer eigenständigen Wissenschaft zu behaupten. Eine Linguistik, die nur innerhalb der "Sprache" bleiben will und nur rein linguistische Einschränkungen anerkennt, gerät aber bezüglich ihrer eigentlichen Zwecke der Beschreibung und Erklärung früher oder später in ein Dilemma, denn je mehr die Linguistik die Sprache abtrennt vom Weltwissen der Sprecher und der Gesellschaft, in der sie leben, um so weniger sind signifikante und relevante Fortschritte in der Beschreibung und Erklärung zu erzielen - auch in der Beschreibung und Erklärung "rein linguistischer" Tatsachen.

Da die strukturalistisch geprägte Linguistik in einer von ihr selbst zu bestimmenden Entfernung von der sprachlichen Praxis arbeitet, entsteht dabei die Gefahr, die kommunikative Praxis zu unterschätzen oder völlig zu vernachlässigen, besonders dann, wenn die Linguisten auch die Rolle des ideal native speaker im Sinne Chomskys einnehmen. Hier wird die Theoriebildung leicht hypertroph und pure Hypothesen werden ohne ausreichende Überprüfung in der Praxis unkritisch angenommen. Viele linguistische Theorien setzen riesige Apparate von hypothetischen Einschränkungen in die Welt, während die praktischen Einschränkungen der realen Kommunikation vernachlässigt werden, in der - wohl richtigen - Vermutung, dass sie keinesfalls alle „rein linguistisch" sind.

Nicht zu Unrecht haben Kritiker aus den eigenen Reihen der Linguistik daher vorgehalten, dass es einer Linguistik, welche die komplexen sozialen Gebrauchsformen von Sprache und die dadurch konstituierten erheblichen Unterschiede in den Funktionen von Sprache außer Acht gelassen hat, an Instrumenten und Begriffen fehlt, die es ermöglichen würden, diese Bereiche mit Aussicht auf Gewinn zu erforschen; vielmehr haben manche der für die Sprache als abstraktes System entwickelten Begrifflichkeiten eine Erforschung der Sprache in ihren Gebrauchszusammenhängen eher verhindert als befördert. Aufgegeben werden sollte vor allem der Gedanke, man könne gleichsam für alle denkbaren Funktionsweisen von Sprache ein einheitliches begriffliches Instrumentarium entwickeln. Wenngleich es sprachliche Grundfunktionen gibt, die in jeder Form des Sprachgebrauchs eine Rolle spielen, können auch sie eine Modifikation erfahren, wenn die Sprache ihre gewohnte Gebrauchsform, die der Mitteilung in der alltäglichen Kommunikation, verlässt. Eine Auseinandersetzung mit der Sprache des Rechts kann und darf sich daher nicht in einer Anwendung andernorts erar- 
beiteter sprachwissenschaftlicher Erkenntnisse auf einen weiteren Gebrauchszusammenhang von Sprache erschöpfen; vielmehr gilt es zu fragen, welche Gemeinsamkeiten und welche Unterschiede zwischen anderen nicht-alltäglichen Formen der Sprachverwendung und denen des juristischen Umgangs mit Sprache bestehen.

\section{Die Komplexität der juristischen Arbeitsweise}

Rechtsnormen sind Gegenstand komplexer institutioneller Auslegungsund Anwendungsverfahren; eine linguistische Beschreibung dieser Rechtstexte kann daher auch nur durch eine Untersuchung der juristischen Arbeitsweisen erfolgen, für die es in der linguistischen Forschung allerdings nur wenige Vorbilder gibt. So lässt sich die Funktionsweise von Normtexten nur sehr bedingt mit einer linguistischen Begrifflichkeit erklären, die für den „Normalfall” der so genannten Alltagskommunikation entwickelt wurde. Normen dienen nicht einfach der Verständigung zwischen zwei Kommunikationspartnern, vielmehr werden sie von vorinformierten und eigens dazu ausgebildeten Experten, die diese Texte bereits kennen, als Mittel komplexer Entscheidungsvorgänge eingesetzt und sind Gegenstand ebenso komplexer, durch institutionelle Regeln und Einflüsse geprägter Arbeitsschritte.

Anders als in der Alltagssprache entfaltet sich die Bedeutung der Normtexte nicht in einfachen Verstehensakten der Rezipienten, sondern in gesteuerten Auslegungsverfahren als Arbeit an und mit Sprache, die institutionsspezifischen Bedingungen unterliegt. Dabei erschließst sich der Gehalt einer Norm erst in einem weit ausgreifenden Netz intertextueller Relationen zu anderen Gesetzestexten, Kommentaren, herangezogenen Urteilen, Gesetzgebungsmaterialien und rechtswissenschaftlichen Aufsätzen und Monographien. Der sich in mehreren Stufen vollziehende Explikationsvorgang setzt für die institutionell korrekte Anwendung eines Gesetzestextes aber nicht nur fachspezifisches Wissen voraus, sondern macht auch Sprachhandlungen notwendig, die sich einer linguistischen Systematisierung entziehen, da sie nicht so sehr sprachlich begründet sind, sondern vor allem auf Zweckmäßigkeitserwägungen zurückgehen. Darüber hinaus ist die Auslegung der Gesetzestexte den Bedingungen der Institutionalität unterworfen, der Einbindung in institutionelle Deutungs- und Arbeitsrahmen, die dem einzelnen Gesetzesanwender in der Praxis meist nur wenig semantischen Spielraum lassen.

Eine weitere Besonderheit ergibt sich aus der anders gearteten Perspektive: während es dem alltagsweltlichen Verständnis von Textinter- 
pretation entspricht, dass der Text bereits vorliegt und erst darauf das Verstehen folgt, geschieht die juristische Arbeit mit Gesetzestexten in der umgekehrten Richtung, nicht vom Normtext zum Fall, sondern vom Fall zum Normtext. Betrachtet man diese Arbeitsrichtung und ihre Konsequenzen für die linguistische Analyse, dann zeigt sich, dass nicht nur die Auslegung eines einzelnen Gesetzestextes semantisch hochkomplex ist, sondern auch die Lösung eines einfachen Rechtsfalles der Vernetzung einer Vielzahl verschiedener Texte bedarf - ein institutionsspezifisches Phänomen, welches indes nur einige wenige Linguisten erkannt haben und das daher erst ansatzweise untersucht ist.

Juristische Auslegung ist mithin weniger eine Interpretation oder Bedeutungsbestimmung im herkömmlichen linguistischen oder alltagssprachlichen Sinn als vielmehr eine Vernetzung von Textstücken, Auslegungsaspekten, Sachverhaltselementen, Zweckerwägungen und rechtspolitischen Überlegungen innerhalb institutioneller Wissensrahmen. Diese institutionellen Eigenschaften der interpretativen Arbeit mit Gesetzestexten aber sind es, die es als fraglich erscheinen lassen, ob die Textualität des Rechts im Allgemeinen und die der Gesetzestexte im Besonderen als sehr spezifischer Fall institutionell wirksamer Fachtexte mit dem normalen und bisher verfügbaren linguistischen Begriffs- und Methodeninventar überhaupt zureichend erfasst und angemessen beschrieben werden können.

\section{Der Satz als die Grenze der Erkenntnis}

Als ihren spezifischen Untersuchungsgegenstand definiert die strukturalistisch geprägte Linguistik das Sprachsystem (langue) und versteht darunter den Sprachbesitz einer Gruppe, das einzelsprachliche System von Elementen und Relationen, kurz: das Regelsystem einer Sprache, das der Sprachverwendung (parole) als der theoretisch unendlichen Menge der konkreten Sprech- und Verstehensakte und den durch sie entstandenen sprachlichen Gebilden zugrunde liegt. Dabei galt der klassischen Linguistik der Satz jahrzehntelang als die oberste linguistische Bezugseinheit: die strukturalistische Linguistik konzentrierte sich fast ausschließlich auf die Analyse und Deskription der Struktur des Satzes, während die generative Transformationsgrammatik ihren Gegenstand, die Sprachkompetenz, als die Fähigkeit des kompetenten Sprechers einer Sprache definierte, eine beliebig große Anzahl von Sätzen zu bilden und zu verstehen, und dabei selbst die Form eines Regelsystems annahm, das die (unendliche) Menge von Sätzen einer Sprache "generieren" soll. 
Erst mit dem Aufkommen der Textlinguistik in den sechziger Jahren ist es zu einer Kritik an dieser Beschränkung linguistischer Forschung auf die Domäne des Satzes gekommen. Es wird geltend gemacht, dass "die oberste und unabhängigste sprachliche Einheit", das "primäre sprachliche Zeichen" nicht der Satz, sondern der Text sei, linguistische Analyse sich somit stärker als bisher auf den Text zu richten habe. Eine prinzipielle Änderung der geltenden sprachtheoretischen Grundlagen ist mit dieser Forderung allerdings nicht verbunden: auch die Textlinguistik versteht sich - wie vorher auch die "Satzlinguistik" - ausdrücklich als eine Linguistik der langue. Die Hierarchie der bis dahin angenommenen Einheiten des sprachlichen Systems (Phonem, Morphem/ Wort, Satzglied, Satz) wird lediglich um die Einheit "Text" erweitert. Darin drückt sich die Auffassung aus, dass nicht nur die Wort- und Satzbildung, sondern auch die Textbildung durch das Regelsystem der Sprache gesteuert wird und auf allgemeinen, sprachsystematisch zu erklärenden Gesetzmäßigkeiten gründet.

Die sprachsystematisch orientierte Textlinguistik hat sich das Ziel gesetzt, diese allgemeinen Prinzipien herauszufinden und systematisch zu beschreiben. Sie rekurriert dabei aber sowohl in theoretisch-begrifflicher als auch in methodischer Hinsicht weitgehend auf Bestimmungen der Satzlinguistik strukturalistischer bzw. generativ-transformationeller Provenienz. Dieser Zusammenhang kommt im Textbegriff besonders deutlich zum Ausdruck, wenn "Text" als eine kohärente Folge von Sätzen definiert wird. Das bedeutet aber, dass der Satz nach wie vor als "Markstein" in der Hierarchie sprachlicher Einheiten angesehen wird; er gilt als die Struktureinheit des Textes.

Wenn die Untersuchung juristischer Texte und des juristischen Umgangs mit Texten unter den Vorzeichen moderner linguistischer Modelle und Methoden eher spärlich erfolgt ist, dann ist dies sicher auch ein Reflex darauf, dass die sprachliche Strukturebene der Texte und Textkonstitution lange Zeit nicht als eigenständige Größe und beschreibenswürdiges Objekt der systematischen Beschäftigung mit Texten anerkannt wurde. Sieht man einmal von den Beiträgen $a b$, welche sich mit der Rechtssprache als Fachsprache und der Verständlichkeit der Rechtssprache beschäftigen, so sind sprachwissenschaftliche Untersuchungen zu den textuellen Bedingungen und Erscheinungen des Rechts erst eine Erscheinung der letzten Jahre.

Das geringe Interesse, welches die Sprachwissenschaft einer Erforschung der Textlichkeit des Rechts auch weiterhin entgegenbringt, liegt allerdings nicht nur an der nach wie vor anhaltenden Dominanz der Beschreibungsebenen der Sätze und Wörter, sondern auch daran, dass gerade die für das Rechtssystem konstituierenden Gesetze in einer Wei- 
se benutzt werden, welche die Struktur dieser Texte in einer oberflächlichen Betrachtungsweise nicht zu einer besonders relevanten Größe macht. Auf den ersten Blick scheint es hier nur auf die einzelnen Paragraphen anzukommen, die in der juristischen Arbeit mit Gesetzen wie in sich abgeschlossene Miniaturtexte behandelt werden; darüber hinaus sind aber auch die Beziehungen zwischen dem einschlägigen Paragraphen und anderen Gesetzesstellen zu beachten, welchen regelmäßig eine wichtige, wenn nicht zentrale Funktion bei der Auslegung des Paragraphentextes oder der Lösung des anstehenden Rechtsfalles zufällt. $\mathrm{Da}$ diese Beziehungen in linguistischen Arbeiten allerdings meist als nebensächlich ausgeblendet werden, kann ihre textuelle Basis, ihr Charakter als innertextuelle oder intertextuelle Relationen und damit ihr genuin sprachlicher Charakter von vielen Sprachwissenschaftlern auch nicht wahrgenommen werden.

\section{Das Kommunikationsmodell der Informatik}

Wenn sich Linguisten mit Texten beschäftigen, lassen sie sich von allgemeinen Konzeptskizzen hinsichtlich des Phänomens Text und der angrenzenden Bereiche leiten. Solche Vorprägungen und Kanalisierungen des Denkens sind ein normales Verwenden tradierten Wissens; derart werden disziplinäre Perspektiven auf einen Untersuchungsgegenstand konstituiert. Angesichts eines interdisziplinär zu erforschenden Gegenstands wie der Sprachlichkeit des Rechts sind einige dieser die Sprachwissenschaft dominierenden Sichtweisen indes kritisch zu hinterfragen.

Nachhaltig geprägt wurde die Sprachwissenschaft vor allem durch die Verwendung von Begriffen aus den "exakten" Wissenschaften, an die sich die durch Chomskys Generativismus angeregte Linguistik zunehmend anlehnte, nachdem die Philologien immer weniger im Stande schienen, aus eigener Kraft neue Fragestellungen und neue Antworten hervorzubringen und auch von der Philosophie, deren letzte große Beiträge zur Sprachtheorie aus der Analytischen und der Ordinary-Language-Philosophie stammen, keine neuen Anstöße zu erwarten waren. Neue Partner suchte und fand die Linguistik in der Nach-ChomskyÄra schließlich in der Psychologie, Biologie und Informatik; dass die Computertechnologie seit den achtziger Jahren so ungeheure Fortschritte machte, erhöhte deren Attraktivität noch weiter und führte nahezu eine ganze Generation von Linguisten in das weitere Feld der Artificial-Intelligence-Forschung, in die Psycholinguistik oder Sprachpsychologie. Seither werden die Grundfragen der Sprachwissenschaft 
in Anlehnung an die Kommunikationsmodelle der Informatik buchstabiert.

Heute erweist sich das Paradigma der Informationstechnologie als enger denn je mit der Theoriebildung über kognitive Prozesse bei Lebewesen verbunden: Wahrnehmen, Auffassen, Begreifen und Verstehen, Verhaltenssteuerung und -ausführung werden von den forschungsstrategisch favorisierten Psycholinguisten in immer größerem Umfang als Prozesse der Informationsverarbeitung modelliert. Angesichts der noch keineswegs an ihre Grenzen gelangten Leistungen der Computertechnologie bietet sich die Modellfunktion der entsprechenden Hard- und Software-Konfigurationen für menschliches kognitives Vermögen immer drängender an. Mit zunehmender Leistungsfähigkeit artifiziell intelligenter Systeme nimmt daher auch die Plausibilität für die Computermetapher zu.

Trotz aller zukünftig zu erwartenden Raffinements stößt die Computermetapher aber an mindestens eine Grenze: die des Verstehens. Das Phänomen des zwischenmenschlichen und Sprach-Verstehens muss komplexer gesehen werden als es die kompliziertesten AI-Modelle bisher vorstellen. Der Grund dafür liegt in erster Linie darin, dass der Bereich der zwischenmenschlichen Interaktion, der Bereich der Wechselwirkungen zwischen kognitiven Systemen im Zusammenhang mit dem Verstehen zusätzlich Beachtung finden muss. Verstehen darf nicht auf psychische Prozesse reduziert, sondern sollte als ein komplexes soziales und kognitives Geschehen zwischen Interaktions- bzw. Kommunikationspartnern begriffen werden. Was die Sprachpsychologie und Psycholinguistik hingegen erforscht, ist nicht das Verstehen als soziales Phänomen, sondern mehr oder weniger ausschließlich das psychologische Phänomen des menschlichen Auffassens und Begreifens, also menschliche Informationserzeugung und -verarbeitung. Wenn die sprachwissenschaftliche Verstehensforschung dessen ungeachtet Verstehen als Informationsverarbeitung konzipiert und von den sozialen Konstituenten des Verstehens nur die Auffassung von Textverarbeitung als auch sozialem und sozial kontextualisiertem Prozess bleibt, wird Verstehen mit Verarbeitung konfundiert.

Soweit die Sprachwissenschaft sich an das Kommunikationsmodell der Informationstechnologie anlehnt, verkürzt sie aber nicht nur das soziale Phänomen des Verstehens auf einen kognitiven Vorgang, auch übernimmt sie mit ihm stillschweigend eine ganze Reihe höchst fragwürdiger Annahmen: so suggeriert und perpetuiert dieses Modell die Vorstellung einer einzigen stabilen und autonomen Wirklichkeit, die wir erkennen, also Stück für Stück „enthüllen”; es suggeriert irreführende und hinsichtlich der Aufgaben einer empirischen Kommunika- 
tionstheorie unfruchtbare "Exaktheit”; es suggeriert, dass die jeweils beteiligten Subjekte als statische Objekte betrachtet werden, deren Verhalten somit unverzerrter Beobachtung zugänglich sei; vor allem aber begünstigt es eine im Verhältnis zur komplexen Kommunikationsrealität an semiotischen Äußerlichkeiten, Oberflächensymptomen und $\mathrm{Zu}$ fälligkeiten orientierte pseudo-empirische Arbeitsweise, da einmal die physikalischen Signale allein im Zentrum stehen, zum anderen "Sender" und „Empfänger" lediglich formal, als ",black boxes" betrachtet werden. Nichts sagt das Modell hingegen über die funktionalen $\mathrm{Zu}$ sammenhänge zwischen kommunikativen Vorgängen und der Struktur der beteiligten Systeme, noch klärt es, wie und warum Kommunikation wirken kann. Kommunikation bleibt damit außerhalb des Systems aller Lebensvorgänge.

\section{Die Bedeutung des Textes für die Kommunikation}

Eine andere, weite Bereiche der Sprachwissenschaft dominierende Perspektive äußert sich darin, dass der Text für gewöhnlich eher als das Ergebnis produktiver sprachlicher Tätigkeit gesehen wird und weniger als der Ausgangspunkt für eine im Prinzip ähnlich aktive und eigentlich auch "produktive" Rezeption. Anders als die Literaturwissenschaft war in der Linguistik von ihren Anfängen als Disziplin an die Vorstellung vorherrschend, dass die Äußerungsproduktion der wichtigere oder der „eigentliche" Teil des Sprachgebrauchs sei. Die Rezeption erscheint damit aber allenfalls als eine Art spiegelbildliche Umkehrung der Produktion. Gestützt wird dieses Denkmuster durch die Auffassung, dass das Herstellen eines Textes, also das Sprechen oder Schreiben, wesentlich darin besteht, Informationen in einen bestimmten Informationsträger, also sprachliche Zeichen in einer bestimmten Anordnung, hineinzupacken. Die Aufgabe des Rezipienten wird dann darin gesehen, möglichst viel von den verpackten Informationen wieder $z u$ "entpacken".

Hinter diesem Konzept des Textes als einer Art Behälter steht die allgemeinere Auffassung, dass sprachliche Zeichen für Gegenstände einer als unabhängig vom Menschen gedachten Wirklichkeit stehen oder wenigstens für Begriffe, die solche wirklichen Gegenstände mehr oder weniger eindeutig und jedenfalls letztlich objektiv abbilden, sodass der sprachliche Prozess im wesentlichen das Finden des "richtigen" Ausdrucks für einen in der Widerspiegelungstätigkeit erarbeiteten Inhalt bzw. des "richtigen" Inhalts für einen gegebenen Ausdruck ist. Wenn es aber eine so klare Beziehung zwischen Zeichen und Bedeutungen 
gibt, dann muss - diesem Denkmuster folgend - die Menge der zu gewinnenden Information in irgendeiner Weise durch das Vorkommen von Zeichen bestimmt sein. Es liegt damit nicht mehr an Bedeutungen vor, als Zeichen vorkommen. Man könnte auch sagen: Mehr Bedeutung als die, die an vorkommende Zeichen gebunden ist, ist linguistisch nicht interessant, ist nicht Gegenstand der Linguistik.

Trotz aller Theorieangebote und ungeachtet der Vielfalt der in der Linguistik kursierenden Modelle zu Verstehen und Verständlichkeit erweisen sich diese Sichtweisen nach wie vor als wirkungsmächtig: wer eine schnelle Definition der Begriffe Verstehen und Verständlichkeit geben möchte, rekurriert auch weiterhin gerne auf die bewährten Schemata. Wie sehr die gängigen disziplinären Sichtweisen der Sprachwissenschaft deren Wahrnehmung bestimmen, kann man daran sehen, dass sie selbst in interdisziplinären Vorhaben immer wieder der gemeinsamen Arbeit zugrunde gelegt werden; dies spiegelte sich auch in ersten Konzeptionsüberlegungen der interdisziplinären Arbeitsgruppe Sprache des Rechts: Vermitteln, Verstehen, Verwechseln an der Berlin-Brandenburgischen Akademie der Wissenschaften wider:

„B Zum Begriff des Verstehens und der Verständlichkeit

B.1 Allgemein

Verstehen': Verstehen ist (in unserem Verständnis im Projekt) ein kognitiver Vorgang der Informationsverarbeitung. Verarbeitet wird sprachlich gegebene und außersprachliche Information. Sprachlich gegeben ist die Information im Text. Außersprachliche Information ist die Kenntnis der Gegebenheiten der jeweiligen Kommunikationssituation (wer, mit wem, wann, wo) und das durch den Text in die Aufmerksamkeit gerückte Weltwissen, letztlich das Weltwissen des ,Durchschnittsverbrauchers'.

Verstehen ist der im Rahmen der Kommunikation komplementäre Vorgang zur Texterzeugung. Die Kommunikation ist erfolgreich, wenn durch die Kommunikation im Wissen des Verstehenden gerade die Vorstellung des Begriffs/Sachverhalts aufgebaut wird, die der Erzeuger vermitteln will. In unserem Beispiel ist das die Vorstellung des Versicherers von den Verpflichtungen und Rechten der Vertragspartner, kurz: die Vorstellung vom Vertrag.

-Verständlichkeit': Der Text des Vertrages ist verständlich in dem Maße, wie durch das Lesen/Studium durch den Durchschnittsverbraucher die intendierte Vorstellung vom Vertrag entsteht, ohne dass der Durchschnittsverbraucher juristisches Expertenwissen über den Vertragstext hinaus einbringt (Kommentare, Gesetzestexte, Rechtswortschatz im allgemeinen)". 


\section{Andere Perspektiven auf den Text und die Kommunikation}

Wenn man sich Kommunikation als komplexen und ganzheitlichen Prozess vorstellt, dann ist das Erscheinen des Textes darin durchaus eine wichtige Phase - wichtig vor allem deshalb, weil in ihr ein materielles Gebilde erscheint. Dennoch bleibt es nur eine Phase in einem sehr viel komplexeren Geschehen. Bevor der Text zu einem wahrnehmbaren Ereignis in unserer Umwelt wird, müssen aber eine Reihe psychischer Prozesse ablaufen, die mit Planung, Kodierung oder auch Auswählen von Wissenselementen nur sehr grob umschrieben sind. Dabei werden gedankliche Gebilde konstruiert und auf vorangegangene oder tradierte, konventionalisierte Erfahrungen mit einer jeweiligen Umgebung bezogen, also ein Kommunikationsgegenstand geschaffen, der den Bedürfnissen und Wünschen des Sprechers entspricht.

Auf der Seite des Rezipienten markiert das Erscheinen des Textes nur einen Anfang. Es werden ebenfalls psychische Prozesse ausgelöst, die mehr sind als ein Identifizieren und Dekodieren vorgefundener sprachlicher Zeichen. Dementsprechend hat man in der Verstehensforschung auch betont, dass der Verstehensprozess konstruktive Momente enthält, dass er nicht nur daten-, sondern auch konzeptgeleitet ist. Die Psycholinguistik hat diese Konstruktionstätigkeit indes sehr schnell unter das Konzept der Wissenskomponenten subsumiert, unter das bloße Gebrauch-Machen von angehäuftem Wissen. Damit entsprach man zwar einem gewohnten Denkmuster, reduzierte aber auch die aktiv werdende Subjektivität auf Wissensunterschiede und Wissensdefizite.

Der Text ist jedenfalls mehr als ein "Informationsbehälter". Auf den Textproduzenten bezogen, repräsentiert er dessen innere Prozesse; für den Rezipienten ist der Text vor allem eine Anregung, ein eigenes gedankliches Gebilde zu schaffen, das dem Text in der aktuellen Situation einen Sinn zuordnet, ihn also für den Rezipienten wichtig macht. Der sinnlich wahrnehmbare Text regt nicht einfach nur dazu an, sich ein Bild von inneren Prozessen und Zuständen des Textproduzenten zu machen oder dessen Bewusstseinsinhalte zu rekonstruieren, wie üblicherweise verkürzend gemeint wird; vielmehr ist der Rezipient gehalten, solche Rekonstruktionen auf die interaktiven Bedingungen zu beziehen, unter denen der Text, also die spezifische Repräsentation, zustande kam. Der Text "transportiert" eben nicht Informationen über eine unabhängige äußere Welt und auch nicht nur über innere Welten der Produzenten, er ist vielmehr ein Produkt des perspektivegeleiteten Zurechtfindens in der Welt, und er dient als Mittel, ein kooperatives 
Zurechtfinden mehrerer Individuen zu orientieren. Er gibt dem Rezipienten eine Hilfestellung (und will ihn natürlich auch beeinflussen und lenken), aber der Rezipient muss selbst aktiv werden.

Des Weiteren muss differenziert werden zwischen dem Kommunikationsmittel Text und dem Kommunikat, das als kognitives Konstrukt einem Text vom Rezipienten zugeordnet wird und das sich folglich von jenen Kommunikaten unterscheidet, die andere Rezipienten dem gleichen Text zuordnen. So undifferenziert der Kommunikat-Begriff zunächst erscheinen mag, weist er doch auf ein grundlegendes Problem hin: auf das Auseinanderfallen des Textinhalts auf dem Weg vom Produzenten zum Rezipienten. Der Textinhalt ist etwas Veränderliches, keine ein für allemal gegebene Konstante, die nur eine Art Abbild eines objektiv gegebenen Tatbestandes wäre. Verschiedene Rezipienten verstehen den gleichen Text unterschiedlich, ja auch bei ein und demselben Rezipienten kann der gleiche Text höchst Unterschiedliches auslösen, wenn er etwa zu verschiedenen Zeiten oder mehrmals selektiv, also mit unterschiedlichen Perspektiven gelesen oder gehört wird. Auch strukturierende Gewichtungen des Inhalts wie das Thema, der Grundgedanke oder die Hauptinformation werden vom Produzenten nur angeboten.

Das Auseinanderfallen des Textinhalts schließt natürlich nicht aus, dass er von einer Reihe überindividuell gültiger Konventionen getragen wird und getragen werden muss. In jeder Sprach- und Kommunikationsgemeinschaft gibt es konventionalisierte Zuordnungen von Zeichen und Bedeutungen, also jene Qualitäten eines Kommunikationsmittels, die in Grammatiken und Wörterbüchern beschrieben sind, die in der Sozialisation angeeignet werden und die einen Grundstock von Referenzherstellungen jedenfalls erleichtern; hinzu kommen Konventionen darüber, wie man verschiedene Texte erzeugt, wozu man sie benutzt, welchen Sinn sie in einer Gemeinschaft haben können. Der Textproduzent bedient sich dieser Konventionen, und der Rezipient kann sie zunächst einmal voraussetzen, sodass insofern weit reichende Verstehenshilfen existieren. Der Rezipient konstruiert sein Kommunikat nicht allein. Allerdings erscheint es fraglich, ob ein Konstrukt wie das der "wörtlichen Bedeutung" für den Text noch brauchbar ist. Eine Annahme, die für die Bausteine eines Textes und sicher auch noch für kurze oder stark konventionalisierte Äußerungen sinnvoll ist, kann ihren Sinn mit zunehmender Länge und Komplexität von Texten verlieren, mit dem dann immer deutlicher werdenden Auseinanderfallen der Textinhalte und dem Zunehmen der Zahl der möglichen Lesarten.

Auch wenn die Zugehörigkeit zur gleichen Sprach- und Kommunikationsgemeinschaft eine Basis für Referenzherstellungen schafft, so 
bringt dies für die Textebene noch nicht sehr viel, denn weitergehende Entscheidungen darüber, was "tatsächlich" gemeint ist und ob etwas "richtig" verstanden wurde, können sich nicht auf „objektive" oder für die ganze Sprachgemeinschaft gültige Kriterien stützen. Streng genommen müsste man davon ausgehen, dass Verstehen als komplexer Prozess zwischenmenschlicher Interaktion im Umgang mit schriftsprachlichen Texten gar nicht möglich ist. Man sollte beim Lesen vielleicht besser davon sprechen, dass man eine Vorstellung oder Idee vom Inhalt oder von der Aussage des Textes, mit anderen Worten eine subjektiv befriedigende Lesart eines Textes gefunden hat. Natürlich kann man die eigene Lesart eines Textes außerdem auch noch für die richtige halten; aber darüber muss man sich - wenn man nicht die Macht hat, die eigene Lesart sozial durchzusetzen bzw. verbindlich zu machen - mit anderen wiederum kommunikativ auseinandersetzen. Die Grenze zwischen Verarbeitung und Verstehen könnte dann markiert werden zwischen Lesartkonstruktion (als kognitivem Prozess des sinnlichen und mentalen Auffassens) einerseits und Lesartvalidierung (als sozialem Interaktions- bzw. Kommunikationsprozess zum Zwecke der Etablierung sozial verträglicher Lesarthierarchien) andererseits.

Aus alledem folgt, dass eine systemorientierte Konzeption des Gegenstands der Rechtslinguistik formuliert werden sollte. Die Einsicht in die Subjektabhängigkeit jeder Erkenntnis muss dazu führen, nicht isolierte Texte zum Thema zu machen, sondern die Prozesse, die an und mit Texten in einer Gesellschaft stattfinden. Letztlich kann erst der Übergang von einer sprachtheoretischen zu einer kommunikationstheoretischen Analyse der Rechtswissenschaft den Zugang zu den sie interessierenden Problemen öffnen. 


\section{Literatur}

Beaugrande, Robert de, Textlinguistik: Zu neuen Ufern? in: Gerd Antos/Heike Tietz (Hrsg.), Die Zukunft der Textlinguistik. Traditionen, Transformationen, Trends. Tübingen 1997, 1-11.

Beaugrande, Robert de/Dressler, Wolfgang Ulrich, Einführung in die Textlinguistik. Tübingen 1981.

Biere, Bernd Ulrich, Verständlich-Machen. Hermeneutische Traditionen Historische Praxis - Sprachtheoretische Begründung. Tübingen 1989.

Brinker, Klaus, Linguistische Textanalyse. Eine Einführung in Grundbegriffe und Methoden. 5. Aufl., Berlin 2001.

Busse, Dietrich, Recht als Text. Linguistische Untersuchungen zur Arbeit mit Sprache in einer gesellschaftlichen Institution. Tübingen 1992.

Busse, Dietrich, Juristische Semantik. Grundfragen der juristischen Interpretationstheorie in sprachwissenschaftlicher Sicht. Berlin 1993.

Busse, Dietrich, Textlinguistik und Rechtswissenschaft, in: Gerd Antos (Hrsg.), Text- und Gesprächslinguistik. Ein internationales Handbuch zeitgenössischer Forschung. Handbücher zur Sprach- und Kommunikationswissenschaft Bd. 16.1. Berlin 2000, 803-811.

Dietrich, Rainer/Klein, Wolfgang (Hrsg.), Sprache des Rechts. Themenheft der Zeitschrift für Literaturwissenschaft und Linguistik 118 (2000).

Dijk, Teun A. van, Textwissenschaft. Eine interdisziplinäre Einführung. Tübingen 1980.

Hartung, Wolfdietrich, Text und Perspektive. Elemente einer konstruktivistischen Textauffassung, in: Gerd Antos/Heike Tietz (Hrsg.), Die Zukunft der Textlinguistik. Traditionen, Transformationen, Trends. Tübingen 1997, 11-25.

Heinemann, Wolfgang/Viehweger, Dieter, Textlinguistik. Eine Einführung. Tübingen 1991.

Kaufmann, Arthur, Recht und Sprache, in: Ders., Beiträge zur juristischen Hermeneutik. Köln 1984, 101-117.

Köck, Wolfram K., Kognition - Semantik - Kommunikation, in: Siegfried J. Schmidt (Hrsg.), Der Diskurs des Radikalen Konstruktivismus, Frankfurt am Main 1987, 340-373.

Kühn, Peter, Juristische Fachtexte, in: Gerhard Helbig (Hrsg.), Deutsch als Fremdsprache. Ein internationales Handbuch zeitgenössischer Forschung. Handbücher zur Sprach- und Kommunikationswissenschaft Bd. 19. Berlin 2001, 582-594.

Luhmann, Niklas, Systeme verstehen Systeme, in: Niklas Luhmann/Karl Eberhard Schorr (Hrsg.), Zwischen Intransparenz und Verstehen. Fragen an die Pädagogik. Frankfurt am Main 1986, 72-117.

Rickheit, Gert/Strohner, Hans, Grundlagen der kognitiven Sprachverarbeitung. Modelle, Methoden, Ergebnisse. Tübingen 1993. 
Rusch, Gebhard, Verstehen verstehen. Ein Versuch aus konstruktivistischer Sicht, in: Niklas Luhmann/Karl Eberhard Schorr (Hrsg.), Zwischen Intransparenz und Verstehen. Fragen an die Pädagogik. Frankfurt am Main 1986, $40-71$.

Rusch, Gebhard, Autopoiesis, Literatur, Wissenschaft. Was die Kognitionstheorie für die Literaturwissenschaft besagt, in: Siegfried J. Schmidt (Hrsg.), Der Diskurs des Radikalen Konstruktivismus, Frankfurt am Main 1987, 374-400.

Rusch, Gebhard, Auffassen, Begreifen und Verstehen. Neue Überlegungen zu einer konstruktivistischen Theorie des Verstehens, in: Siegfried J. Schmidt (Hrsg.), Kognition und Gesellschaft. Der Diskurs des Radikalen Konstruktivismus 2. Frankfurt am Main 1992, 214-256.

Saussure, Ferdinand de, Grundfragen der allgemeinen Sprachwissenschaft. 2. Aufl., Berlin 1967.

Scherner, Maximilian, Sprache als Text. Ansätze zu einer sprachwissenschaftlich begründeten Theorie des Textverstehens. Forschungsgeschichte - Problemstellung - Beschreibung. Tübingen 1984.

Schnotz, Wolfgang, Aufbau von Wissensstrukturen. Untersuchungen zur Kohärenzbildung bei Wissenserwerb mit Texten. Weinheim 1994.

Schmidt, Siegfried J., Kognitive Autonomie und soziale Orientierung. Konstruktivistische Bemerkungen zum Zusammenhang von Kognition, Kommunikation, Medien und Kultur. Frankfurt am Main 1994. 